\title{
Identifying pathway modules of tuberculosis in children by analyzing multiple different networks
}

\author{
LU CHENG, YULING HAN, XIUXIA ZHAO, XIAOLI XU and JING WANG \\ Department of Respiratory Medicine, Qilu Children's Hospital of Shandong University, \\ Jinan, Shandong 250022, P.R. China
}

Received July 12, 2017; Accepted October 13, 2017

DOI: $10.3892 /$ etm.2017.5434

\begin{abstract}
Tuberculosis (TB), which is caused by the mycobacterium TB, is the major cause of human death worldwide. The aim of this study was to identify the biomarkers involved in child TB. Gene expression data were obtained from the Array Express Archive of Functional Genomics Data. Gene expression data and protein-protein interaction (PPI) data were downloaded to construct differential gene co-expression networks (DCNs). The Benjamini-Hochberg algorithm was used to correct the P-value. In total, 3,820 edges (PPIs) and 1,359 nodes (genes) were obtained from the human-related PPIs data and gene expression data at the criteria of absolute value of Pearson's correlation coefficient $>0.8$. The DCNs were formed by these edges and nodes. Thirteen seed genes were obtained by ranging z-scores. Eight significant multiple different modules were identified from DCNs using the statistical significant test. In conclusion, the seed genes and significant modules constitute potential biomarkers that reveal the underlying mechanisms in child TB. The new identified biomarkers may contribute to an understanding of TB and provide a new therapeutic method for the treatment of TB.
\end{abstract}

\section{Introduction}

Tuberculosis (TB), which is caused by mycobacterium TB, is a major cause of human mortality worldwide, with two million

Correspondence to: Dr Lu Cheng, Department of Respiratory Medicine, Qilu Children's Hospital of Shandong University, 23976 Jingshi Street, Jinnan, Shandong 250022, P.R. China

E-mail: chenglumed@163.com

Abbreviations: WHO, World Health Organization; BCG, Bacille Calmette-Guerin; NAAT, nucleic acid amplification test; PPI, protein-protein interaction; GCN, gene co-expression network; DCN, differential gene co-expression network; M-DM, multiple differential module; RMA, robust multichip average; GGI, gene-gene functional interaction; NAIC, North American Indian childhood cirrhosis; ASO, antisense oligonucleotide; TB, tuberculosis

Key words: differential gene co-expression networks, multiple differential modules, protein-protein interactions, tuberculosis deaths and ten million new cases of TB occurring annually (1). Children are more susceptible to the infection of mycobacterium TB due to their having a relatively weaker immune system compared with adults $(2,3)$. The World Health Organization (WHO) reported that almost one million children were infected with the mycobacterium TB in 2015 (4). India, Indonesia, China, Nigeria, Pakistan and South Africa account for $60 \%$ of newly identified cases (5). There are more than 30,000 new children cases of multidrug-resistant TB in 2015 worldwide (6).

Vaccination with BacilleCalmette-Guerin (BCG) is an effective form of prevention of TB. The BCG vaccine has $60-80 \%$ protective effect against severe types of TB in children, especially meningitis (7). The Xpert Mycobacterium tuberculosis/rifampicin (MTB/RIF) assay can be used to diagnose TB and yield reliable results. Zar et al reported that Xpert MTB/RIF was a useful assay for the rapid and reliable diagnosis of paediatric TB in African children, using induced sputum and nasopharyngeal as the specimens (8). Gous et al also used the Xpert MTB/RIF assay to diagnose TB in childhood (9). Fiebig et al used the nucleic acid amplification tests and culture of gastric aspirates to detect bacteriological confirmation of TB in German children. Those authors found that the combined use of molecular assay and culture method had an improved test accuracy rate (10).

Protein-protein interactions (PPI) play an important role in all biological processes. The interaction networks can be used to explore the intricate protein organizations and cellprocesses $(11,12)$. Safaei et al carried out a PPI network study on cirrhosis liver disease. Authors of that study found that the regulation of cell survival and lipid metabolism were pivotal biological processes in cirrhosis disease (13). In ovarian cancer, 12-gene network modules have been identified using the differential co-expression PPI network. The gene expression data and PPI networks can be used to develop effective biomarkers for understanding disease mechanisms (14). Ramadan et al combined the PPI and gene co-expression network (GCN) to analyze breast cancer (15).

In the present study, the PPI network and GCN were employed to analyze the latent and active period of TB in children. Thirteen seed genes were found in the differential gene co-expression networks (DCNs), and eight multiple differential modules (M-DMs) were identified based on the DCNs (16). The identified M-DMs provided new insights into the development of TB in children. 


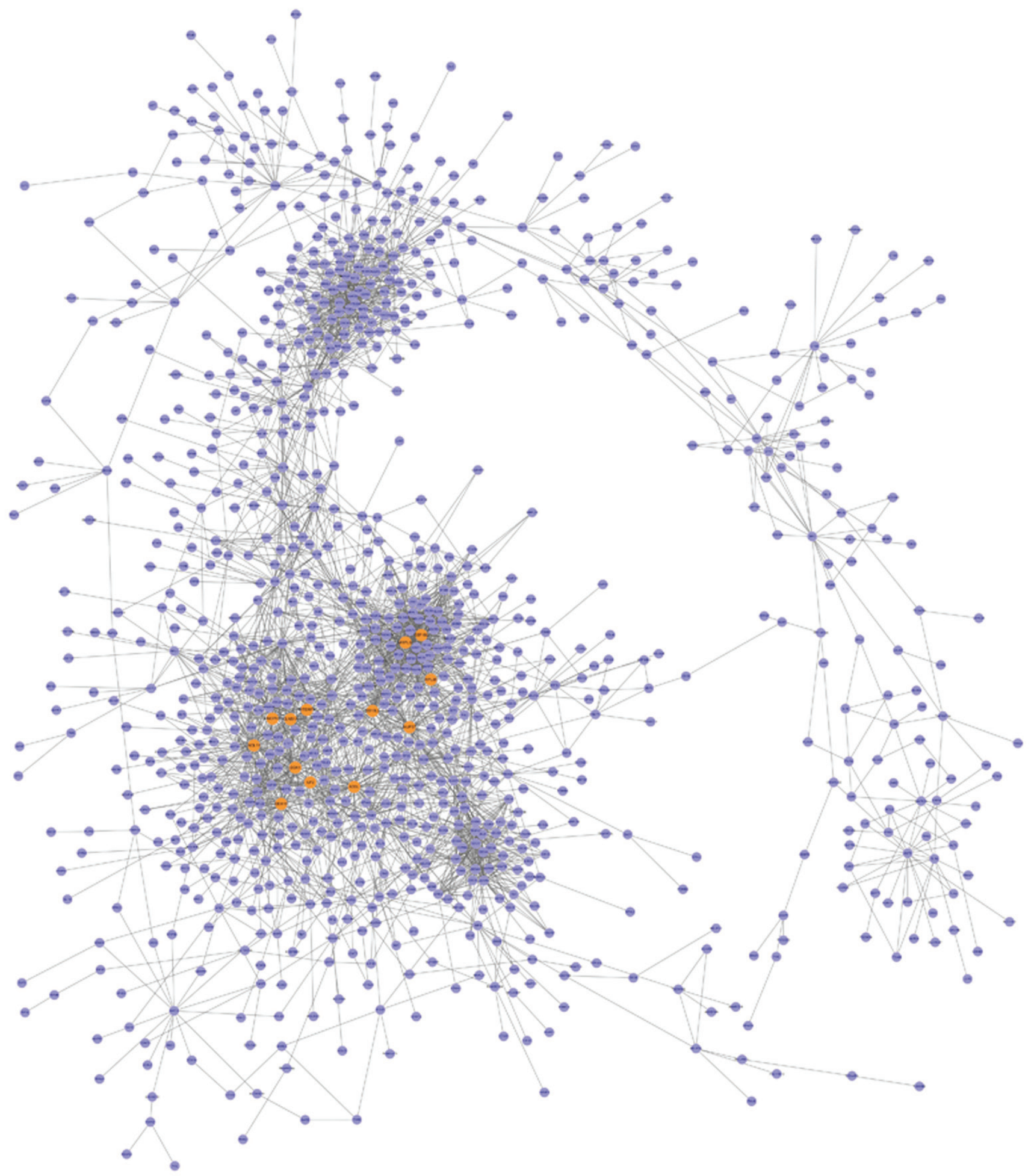

Figure 1. The DCNs contained 3,820 edges (PPIs) and 1,359 nodes (genes). DCNs, differential gene co-expression networks; PPI, protein-protein interaction.

\section{Materials and methods}

Gene expression data. The Array Express Archive of Functional Genomics Data is a functional genomics database at the European Bioinformatics Institute. The microarray data of E-GEOD-39940 were downloaded from the Array Express database. The data contained the gene expression profilings of patients who were HIV-negative, suffered from latent period of TB $(n=54)$ and active period of TB $(n=70)$.
In order to eliminate the influence of non-specific hybridization, the robust multichip average method was used to correct background. The quantile-based algorithm was carried out to normalize the data. The probes were discarded when they did not match any genes. In total, 13,997 genes were obtained after the mapping between gene IDs and probe IDs.

PPI data. Human related PPI data were obtained from the The Search Tool for the Retrieval of Interacting database, 
containing 787,896 pairs and 16,730 genes. The genes that were included in gene expressions and PPIs were selected to construct DCN. After processing, 501,736 PPI pairs and 12,310 genes were obtained.

Construction of DCNs. The absolute value of the Pearson's correlation coefficient of PPI pairs of the active TB samples were calculated. The PPIs were selected if the corresponding absolute value was $>0.8$. Finally, 3,820 edges (PPIs) and 1,359

$$
w_{i, j}= \begin{cases}\frac{\left(\log p_{i}+\log p_{j}\right)^{1 / 2}}{\left(2 * \max _{\mathrm{t} \in \mathrm{V}}\left|\log \mathrm{p}_{\mathrm{t}}\right|\right)^{1 / 2}}, & \text { if } \operatorname{cor}(\mathrm{i}, \mathrm{j}) \geq \delta, \\ 0, & \text { if } \operatorname{cor}(\mathrm{i}, \mathrm{j})<\delta,\end{cases}
$$

nodes (genes) were obtained to construct the DCNs.

The one-tailed t-test was used to calculate the P-value of differentially expressed genes in the latent and active TB. The weight value of each interaction was calculated based on the P-values of genes according to EdgeR (17) as follows:

Where pi and pj are the P-values of the differential expression of gene $\mathrm{i}$ and gene $\mathrm{j}$, respectively. $\mathrm{V}$ is the node set of the co-expression network. In addition, cor(i,j) indicates the absolute value of Pearson's correlation between gene $i$ and $j$.

$$
g(i)=\sum_{j \in N(i)} A_{i j}^{\prime} g(j)
$$

Construction of M-DMs. The construction of M-DMs consists of three steps: i) Seed genes prioritization, ii) module search based on each gene, and iii) the refinement of candidate modules. i) The importance of each gene in the networks was calculated as:

where $g(i)$, the importance of vertex $i$ in the network; $N(i)$, the adjacent set of gene $\mathrm{i} ; \mathrm{A}^{\prime}$, the degree normalized weighted adjacent set, which is calculated as $\mathrm{A}^{\prime}=\mathrm{D}^{-1 / 2} \mathrm{AD}^{1 / 2}$, where $\mathrm{D}$ is the diagonal set of A.

The $\mathrm{g}(\mathrm{i})=\mathrm{z}$-score, and the genes were then ranked by the $\mathrm{z}$-scores. The genes with the highest $1 \% \mathrm{z}$-scores were selected as the seed genes. ii) For each seed gene $v \in V$, it was selected as one differential module $\mathrm{C}$. Then the gene $\mathrm{u}$, which was adjacent to the gene $\mathrm{v}$ in the network was incorporated into this module, designated as module $\mathrm{C}^{\prime}$. The entropy change of the two modules was assessed as: $\Delta \mathrm{H}\left(\mathrm{C}^{\prime}, \mathrm{C}\right)=\mathrm{H}\left(\mathrm{C}^{\prime}\right)-\mathrm{H}(\mathrm{C})$.

$\Delta \mathrm{H}\left(\mathrm{C}^{\prime}, \mathrm{C}\right)>0$ exhibited that the connectivity of module $\mathrm{C}$ was increased by the joining of gene $u$. This was then joined to the adjacent gene $\mathrm{u}$, which potentially increased the $\Delta \mathrm{H}$ in module $\mathrm{C}$ until the $\Delta \mathrm{H}$ was no longer able to increase. iii) The candidate module was removed if it contained $<5$ nodes. If the overlapping degree between two modules was $\geq 0.5$, the two modules were merged into one module.

The statistical significant test of candidate M-DMs. In total, 3,820 edges were selected randomly from 501,736 edges and formed the random network. The module searching was carried out following the above mentioned steps. The random networks were constructed 100 times, and 2,318 modules
Table I. Genes with highest $1 \%$ z-scores in DCNs were selected as the seed genes ${ }^{\mathrm{a}}$.

\begin{tabular}{ll}
\hline Gene name & z-score \\
\hline SS18L2 & 473.111 \\
NOL11 & 457.7947 \\
ADSL & 438.8713 \\
ILF2 & 365.7652 \\
DDX18 & 345.6201 \\
DDX1 & 330.0789 \\
CLNS1A & 306.1616 \\
ENOPH1 & 300.3362 \\
MTERF3 & 300.3337 \\
MRPL32 & 294.2793 \\
NUP37 & 287.2869 \\
RPL35 & 285.7214 \\
EEF1B2 & 284.5787
\end{tabular}

an total, 13 seed genes were obtained.

Table II. The P-value of 11 candidate M-DMs was calculated using the Benjamini-Hochberg algorithm ${ }^{\mathrm{a}}$.

\begin{tabular}{lcc}
\hline Modules & P-values & Entropy \\
\hline 1 & 0 & 0.847 \\
2 & 0 & 0.687 \\
3 & 0 & 0.739 \\
5 & 0 & 0.721 \\
6 & 0 & 0.775 \\
7 & 0 & 0.851 \\
11 & 0 & 0.798 \\
12 & 0 & 0.716 \\
\hline
\end{tabular}

${ }^{\mathrm{a}} \mathrm{P} \leq 0.05$ was considered statistically significant.

were constructed. The empirical P-value of the candidate module was calculated as the probability of the module, which has the observed score or smaller score by chance. The Benjamini-Hochberg algorithm was used to correct the P-value (16). The modules that had the P-value of $\leq 0.05$ were selected as the differential modules.

\section{Results}

Construction of DCNs. The human-related PPI and gene expression data were downloaded to construct the DCNs. Based on the criteria of absolute value of Pearson's correlation coefficient $>0.8,3,820$ edges (PPIs) and 1,359 nodes (genes) were obtained (Fig. 1). The DCNs consisted of these edges and nodes.

Identification of candidate $M-D M s$. The genes which had the highest $1 \% \mathrm{z}$-scores in DCNs were selected as the seed genes. On aggregate, 13 seed genes were obtained (Table I). 
A

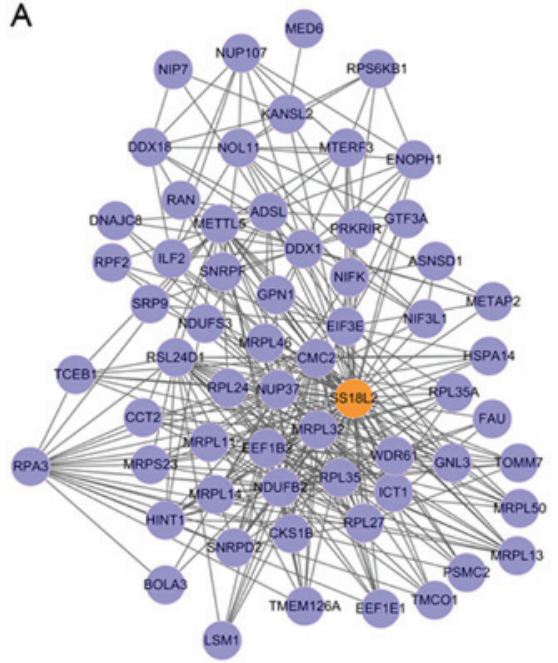

C

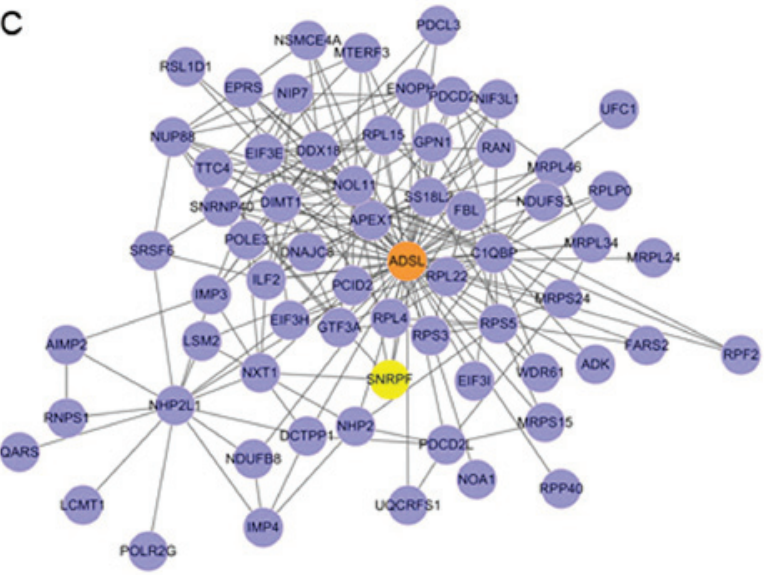

E

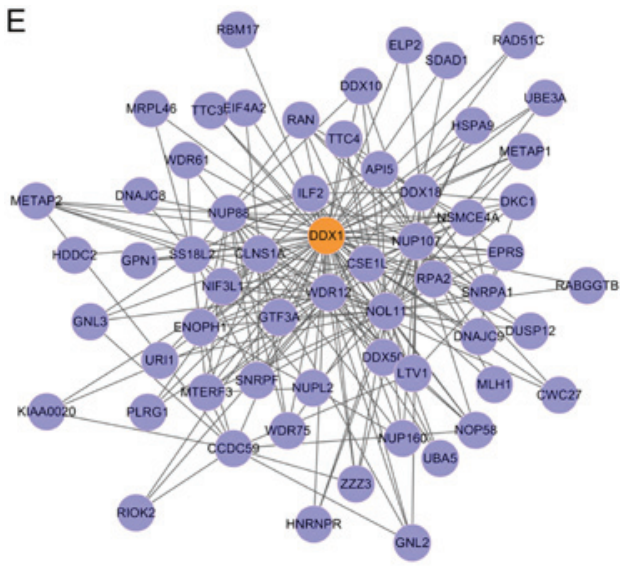

G

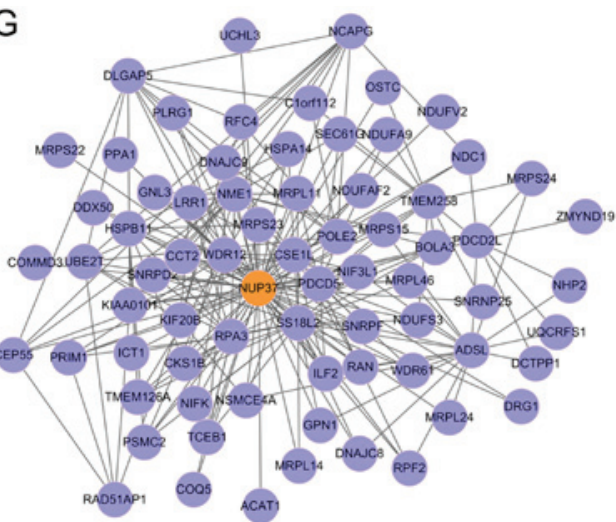

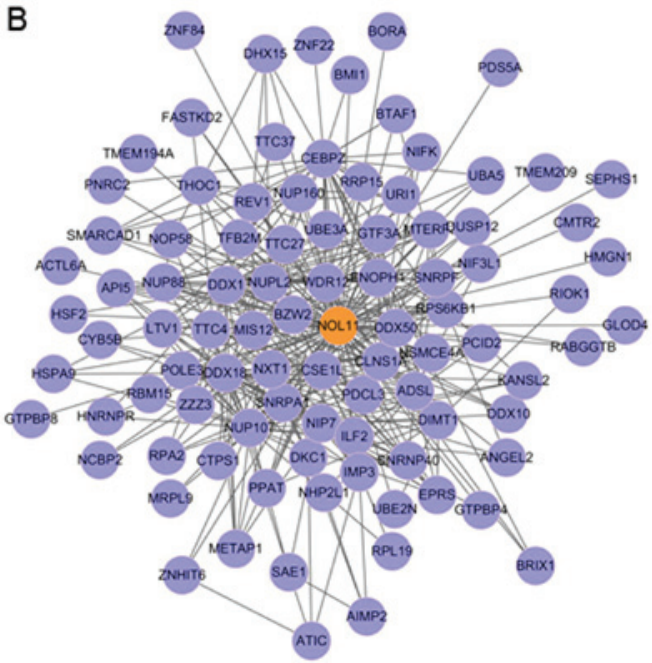
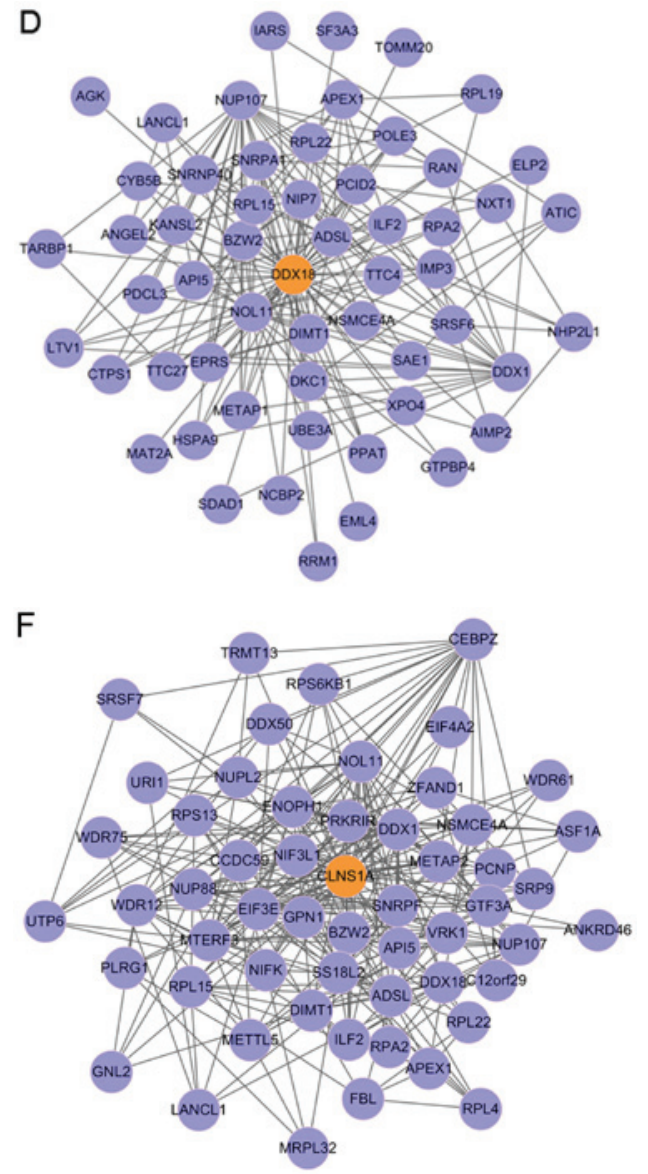

$\mathrm{H}$

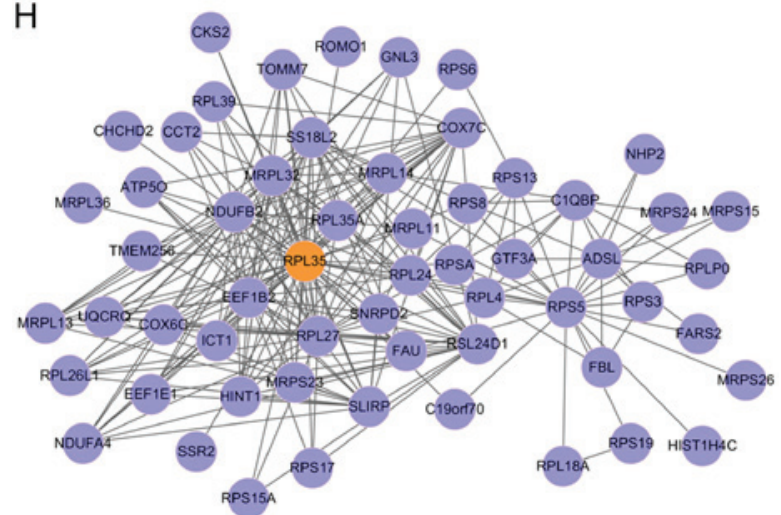

Figure 2. The M-DMs identified from the DCNs. (A-H) The 8 M-DMs, respectively, are presented. M-DMs, multiple differential modules; DCNs, differential gene co-expression networks. 
The z-scores ranged from 284.5787 to 473.111 . The seed genes contained SS18L2, NOL11, ADSL, ILF2, DDX18, DDX1, CLNS1A, ENOPH1, MTERF3, MRPL32, NUP37, RPL35 and $E E F 1 B 2$. After the modules were investigated and refined, 11 modules were obtained.

Identification of candidate M-DMs. The P-value of the 11 candidate M-DMs were calculated and corrected using the Benjamini-Hochberg algorithm. The modules with $\mathrm{P} \leq 0.05$ were regarded as the objective modules. Finally, 8 modules were selected as significant differential modules (Table II and Fig. 2). The module entropy ranged from 0.687 to 0.851 .

\section{Discussion}

From a systematic biology point of view, diseases are caused by the fluctuations to the gene expression network. Such fluctuations change significantly during the disease progressions (18). Schwarz et al combined the PPI works and expression genes to examine the biological processes and genes related with schizophrenia (19). The PPI and gene-gene functional interaction networks were constructed to identify potential biomarkers of pediatric adreno cortical carcinoma (20).

In the present study, we introduced a new method based on M-DMs to identify new biomarkers to better understand the molecular mechanisms and search for potential biomarkers of TB. We identified 8 modules associated with TB.

Humans possess two SS18 homologous genes, SS18L1 and SS18L2. The SS18L2 gene has three exons and is mapped to chromosome 3, with band p21 (21). de Bruijn reported that SS18 encoded nuclear proteins and functioned as a transcriptional co-activator. The fusion of either $S S X$ genes or $S S 18$ is a hallmark of human synovial sarcoma (22).

Nuclear protein $11(N O L 11)$ is a metazoan-specific protein and is involved in ribosome biogenesis. NOL11 also plays an important role in the maturation of 18S RNA and pathogenesis of North American Indian childhood cirrhosis (23).

Human adenylosuccinatelyase $(A D S L)$ is a bifunctional enzyme acting in two pathways of purine nucleotide metabolism including de novo purine synthesis and purine nucleotide recycling (24). The human liver $A D S L$ gene was cloned and mapped to chromosome $22(25,26)$.

The antisense oligonucleotides (ASOs) combine with RNA to form heteroduplexes, which can be specifically recognized by the interleukin enhancer-binding factor 2 and 3 complex (ILF2/3). The combination of ASO and $I L F 2 / 3$ modulates gene expression by alternative splicing (27). ILF2 mRNA accumulates in the pachytene spermatocytes. ILF2 is also expressed in the adult ovary and different embryo tissues (28).

DEAD-Box Helicase 1(DDXI) was found in a high-molecular complex containing a series of Drosha-associated polypeptides (29). Low $D D X 1$ levels are associated with poor clinical outcome in serious ovarian cancer by the cancer genome atlas and $D D X 1$ plays an important role in the modulation of miRNA maturation (30).

Nevertheless, there are some drawbacks to the present study. The study included 124 samples, which is not a sufficient amount of samples to support the conclusions and future studies are to be conducted to confirm the findings. In addition, the results were not verified by clinical experiments.
In conclusion, in the present study, we identified 8 significant different modules using the new bioinformatic methods. We believe that the present study will benefit the understanding of TB in children and provide new therapeutic methods to combat the disease.

\section{References}

1. Rawat J, Sindhwani G and Juyal R: Clinico-radiological profile of new smear positive pulmonary tuberculosis cases among young adult and elderly people in a tertiary care hospital at Deheradun (Uttarakhand). Indian J Tuberc 55: 84-90, 2008.

2. Starke JR: Resurgence of tuberculosis in children. Pediatr Pulmonol Suppl 11: 16-17, 1995.

3. Smith S, Jacobs RF and Wilson CB: Immunobiology of childhood tuberculosis: A window on the ontogeny of cellular immunity. J Pediatr 131: 16-26, 1997.

4. Yoo AS,StaahlBT,ChenLand Crabtree GR:MicroRNA-mediated switching of chromatin-remodelling complexes in neural development. Nature 460: 642-646, 2009.

5. World Health Organisation (WHO): Global health observatory data: (2017). doi: http://www.who.int/gho/hiv/en/.

6. Hamilton CD, Swaminathan S, Christopher DJ, Ellner J, Gupta A, Sterling TR, Rolla V, Srinivasan S, Karyana M, Siddiqui S, et al: RePORT International: Advancing tuberculosis biomarker research through global collaboration. Clin Infect Dis 61: 155-159, 2015.

7. Trunz BB, Fine P and Dye C: Effect of BCG vaccination on childhood tuberculous meningitis and miliary tuberculosis worldwide: A meta-analysis and assessment of cost-effectiveness Lancet 367: 1173-1180, 2006.

8. Zar HJ, Workman L, Isaacs W, Dheda K, Zemanay W and Nicol MP: Rapid diagnosis of pulmonary tuberculosis in African children in a primary care setting by use of Xpert MTB/RIF on respiratory specimens: A prospective study. Lancet Glob Health 1: 97-104, 2013.

9. Gous N, Scott LE, Khan S, Reubenson G, Coovadia A and Stevens W: Diagnosing childhood pulmonary tuberculosis using a single sputum specimen on Xpert MTB/RIF at point of care. S Afr Med J 105: 1044-1048, 2015.

10. Fiebig L, Hauer B, Brodhun B, Balabanova $Y$ and Haas W: Bacteriological confirmation of pulmonary tuberculosis in children with gastric aspirates in Germany, 2002-2010. Int J Tuberc Lung Dis 18: 925-930, 2014.

11. Stelzl U, Worm U, Lalowski M, Haenig C, Brembeck FH, Goehler H, Stroedicke M, Zenkner M, Schoenherr A, Koeppen S, et al: A human protein-protein interaction network: A resource for annotating the proteome. Cell 122: 957-968, 2005.

12. Ferrari R, Forabosco P, Vandrovcova J, Botía JA, Guelfi S, Warren JD, Momeni P, Weale ME, Ryten M and Hardy J: UK Brain Expression Consortium (UKBEC): Frontotemporal dementia: Insights into the biological underpinnings of disease through gene co-expression network analysis. Mol Neurodegener 11: 21, 2016.

13. Safaei A, Rezaei Tavirani M, Arefi Oskouei A,Zamanian Azodi M, Mohebbi SR and Nikzamir AR: Protein-protein interaction network analysis of cirrhosis liver disease. Gastroenterol Hepatol Bed Bench 9: 114-123, 2016.

14. Jin N, Wu H, Miao Z, Huang Y, Hu Y, Bi X, Wu D, Qian K, Wang L, Wang C, et al: Network-based survival-associated module biomarker and its crosstalk with cell death genes in ovarian cancer. Sci Rep 5: 11566, 2015.

15. Ramadan E, Alinsaif S and Hassan MR: Network topology measures for identifying disease-gene association in breast cancer. BMC Bioinformatics 17: 274, 2016.

16. Feser WJ, Fingerlin TE, Strand MJ and Glueck DH: Calculating Average Power for the Benjamini-Hochberg Procedure. J Stat Theory Appl 8: 325-352, 2009.

17. Robinson MD, McCarthy DJ and Smyth GK: edgeR: A bioconductor package for differential expression analysis of digital gene expression data. Bioinformatics 26: 139-140, 2010.

18. MaX, Gao L, Karamanlidis G, Gao P,Lee CF, Garcia-Menendez L, Tian R and Tan K: Revealing pathway dynamics in heart diseases by analyzing multiple differential networks. PLOS Comput Biol 11: e1004332, 2015.

19. Schwarz E, Izmailov R, Liò P and Meyer-Lindenberg A: Protein interaction networks link schizophrenia risk loci to synaptic function. Schizophr Bull 42: 1334-1342, 2016. 
20. Kulshrestha A, Suman S and Ranjan R: Network analysis reveals potential markers for pediatric adrenocortical carcinoma. Onco Targets Ther 9: 4569-4581, 2016.

21. de Bruijn DR, Kater-Baats E, Eleveld M, Merkx G and Geurts Van Kessel A: Mapping and characterization of the mouse and human SS18 genes, two human SS18-like genes and a mouse Ss18 pseudogene. Cytogenet Cell Genet 92: 310-319, 2001.

22. de Bruijn DR, Allander SV, van Dijk AH, Willemse MP, Thijssen J, van Groningen JJ, Meltzer PS and van Kessel AG: The synovial-sarcoma-associated SS18-SSX2 fusion protein induces epigenetic gene (de)regulation. Cancer Res 66: 9474-9482, 2006.

23. Freed EF, Prieto JL, McCann KL, McStay B and Baserga SJ: NOL11,implicated in the pathogenesis of North American Indian childhood cirrhosis, is required for pre-rRNA transcription and processing. PLoS Genet 8: e1002892, 2012.

24. Kmoch S, Hartmannová H, Stibůrková B, Krijt J, Zikánová M and Sebesta I: Human adenylosuccinate lyase (ADSL), cloning and characterization of full-length cDNA and its isoform, gene structure and molecular basis for ADSL deficiency in six patients. Hum Mol Genet 9: 1501-1513, 2000.

25. Stone RL, Aimi J, Barshop BA, Jaeken J, Van den Berghe G, Zalkin $\mathrm{H}$ and Dixon JE: A mutation in adenylosuccinate lyase associated with mental retardation and autistic features. Nat Genet 1: 59-63, 1992.
26. Fon EA, Demczuk S, Delattre O, Thomas G and Rouleau GA: Mapping of the human adenylosuccinate lyase (ADSL) gene to chromosome 22q13.1->q13.2. Cytogenet Cell Genet 64: 201-203, 1993.

27. Rigo F, Hua Y, Chun SJ, Prakash TP, Krainer AR and Bennett CF: Synthetic oligonucleotides recruit ILF2/3 to RNA transcripts to modulate splicing. Nat Chem Biol 8: 555-561, 2012.

28. López-Fernández LA, Párraga $M$ and del Mazo J: Ilf2 is regulated during meiosis and associated to transcriptionally active chromatin. Mech Dev 111: 153-157, 2002.

29. Gregory RI, Yan KP, Amuthan G, Chendrimada T, Doratotaj B, Cooch N and Shiekhattar R: The microprocessor complex mediates the genesis of microRNAs. Nature 432: 235-240, 2004.

30. Han C, Liu Y, Wan G, Choi HJ, Zhao L, Ivan C, He X, Sood AK, Zhang $\mathrm{X}$ and $\mathrm{Lu} \mathrm{X}$ : The RNA-binding protein DDX1 promotes primary microRNA maturation and inhibits ovarian tumor progression. Cell Reports 8: 1447-1460, 2014.

(i) $(9)$ This work is licensed under a Creative Commons Attribution-NonCommercial-NoDerivatives 4.0 International (CC BY-NC-ND 4.0) License. 